\title{
Aprovechamiento forestal maderable en cuatro municipios del departamento de Chocó, Colombia
}

\section{Timber forestry in four municipalities in the department of Chocó, Colombia}

\author{
Exploração madeireira em quatro municípios \\ no departamento de Chocó, Colômbia
}

\author{
Melida Martínez Guardia1, Jhon Jerley Torres Torres² \& Henry Hernan Medina Arroyo³ \\ 'Zootecnista, Especialista en Informática Educativa, Magister en Ciencia de \\ Reproducción Animal, Doctora en Ciencia Animal. ${ }^{2}$ Ingeniero Agroforestal. \\ ${ }^{3}$ Ingeniero Agroforestal, Magister en Manejo de Bosque. \\ 1,2,3Grupo de Investigación Ciencia Animal y Recursos Agroforestales (CARA), Programa de \\ Ingeniería Agroforestal, Universidad Tecnológica del Chocó “Diego Luis Córdoba”, Quibdó. Colombia. \\ 1melidamaguar@yahoo.es, ${ }^{2}$ jhonjerleytorres@gmail.com, ${ }^{3}$ hehemear@yahoo.com
}

\section{Resumen}

Se caracterizó el aprovechamiento de los productos forestales maderables de cuatro municipios del departamento del Chocó, Colombia, teniendo en cuenta los procesos de transformación, uso y comercialización. Para la recolección de la información, se formularon encuestas estructuradas, las cuales fueron aplicadas a los aserradores y ebanistas; paralelo a esto, se realizaron visitas al bosque donde se observaron los procesos productivos. Como resultado se obtuvo que las especies maderables más aprovechadas en los municipios de Atrato, Cértegui, Istmina y Medio San Juan son: Brosimum_utile, Hymenaea oblongifolia, Pseudolmedia laevigata, Cedrela odorata, Couma macrocarpa, Calophyllum longifolium y Humiriastrum procerum. Los rendimientos promedios en la cosecha del bosque son $1,72 \mathrm{~m}^{3}$ hora $^{-1}$, equivalente a $412 \mathrm{~m}^{3}$ $\mathrm{mes}^{-1}$, de los cuales se obtienen bloques y trozas. El $69 \%$ de este material es comercializado con intermediario y el $31 \%$ restante es vendido a los centros de acopios locales, quienes lo transforman en 2x2, Guayacanes, 2x3, vigas, tablas y soleras. Los desperdicios generados de las labores de aprovechamiento, en casi su totalidad no son aprovechados a excepción del aserrín, del cual una parte se utiliza en labores agropecuarias y el resto es arrojado a los ríos. Producto del segundo grado de transformación a nivel local se obtienen: camas, sillas, canaletes, canoas, entre otros materiales. Las actividades de cosecha del bosque en los cuatros municipios en estudio se caracterizan porque: son realizadas con motosierra, la madera obtenida es transportada a hombro, balsas (fluvialmente) y utilizando tracción animal. Referente a la relación beneficio costo, se obtuvo un valor de 6 .

Palabras clave: aprovechamiento forestal, Chocó, especies maderables, tala selectiva, transformación

\section{Abstract \\ The use of the timber-yielding forest products from four municipalities of the department of Cho- có, Colombia was characterized, considering the processes of transformation, use and commer- cialization. The gathering of information, was ca- rried out by the formulation of structured surveys, which were applied to the sawyers and woodwor-}


kers; in parallel, visits to the forest were conducted where the productive processes were observed. As a result, the most exploited timber species in the municipalities of Atrato, Cértegui, Istmina y Medio San Juan were identified: Brosimum utile, Hymenaea oblongifolia, Pseudolmedia laevigata, Cedrela odorata, Couma macrocarpa, Calophyllum longifolium y Humiriastrum procerum. The average yields in the harvest of the forest are 1,72 $\mathrm{m}^{3}$ hora-1 $^{-1}$, equivalently to $412 \mathrm{~m}^{3} \mathrm{mes}^{-1}$, which blocks and troza are obtained. $69 \%$ of this material is commercialized by intermediary and the remainder $31 \%$ is sold to local collection centers, where it is transformed in $2 \times 2$, Lignum vitae, $2 \times 3$, beams, stage and crossbeams. The waste generated in the productive processes are not used with the exception of the sawdust, of which a part of this is used in farm work, and the rest is thrown into the rivers. Product of the second degree of transformation at the local level as: beds, chairs, canaletes, canoes, among other material obtained. The activities of harvest of the forest in the four municipalities in study are characterized because: they are made with a chainsaw, timber is transported over the shoulder, rafts (river navigation) and using animal traction. Concerning the benefit cost relationship, a value of 6 was obtained.

Key-words: forestry, Chocó, timber species, selective logging, transformation

\section{Resumo}

Caracterizou-se o aproveitamento dos produtos florestais madeireiros de quatro municípios do estado de Chocó, Colômbia, levando em consideração os processos de transformação, uso e comercialização. Para a coleta de informação, foram formuladas entrevistas estructuradas que foram aplicadas aos carpinteiros e ebanistas. Concomitantemete foram feitas visitas ao bosque onde se observaram os processos produtivos. Como resultado se obteve que as espécies madeireiras mais aproveitadas nos municípios de Atrato, Cértegui, Istmina e Medio San Juan são: A produtividade media na colheita do bosque corresponde a 1,72 m3 hora-1, equivalente a $412 \mathrm{~m} 3$ mes-1, sendo que desta é obtido blocos e trozas. O $69 \%$ dese material é comercializado com intermediário e o $31 \%$ restante é vendido aos centros de acopio locais onde o transformam. Os esperdícios gerados nas labores de aproveitamento não são 100\% aproveitadas, exceto a serrage, da qual uma parte se utiliza em labores agropecuarias e o restante é jogado nos rios. Produto do segundo grau de transformação a nível local obtemse: camas, cadeiras, canaletes, canoas, entre outros materiais. As atividades de colheita do bosque nos quatros municípios estudados se caracterizam porque são realizadas con motoserra, a madeira é obtida e transportada no ombro, fluvialmente e utilizando tração animal. Referente à relação beneficio - custo se obteve um valor de 6 .

Palavras-chave: silvicultura, Choco, espécies de madeira, extração seletiva, processamento

\section{Introducción}

Los bosques tropicales, se caracterizan por poseer una gran riqueza florística y faunística, por lo que son considerados grandes almacenes que albergan una amplia variedad de productos forestales maderables y no maderables, cuyo valor es inmensurable, especialmente para la economía de los países en desarrollo, dado que durante siglos, la población rural ha obtenido su subsistencia de estos ecosistemas, sobre todo, por la recolección y uso de los productos forestales maderables allí presentes (González, 2003; Arias, 2007; Challenger \& Soberón, 2008; Guariguata et al., 2009). Paralelo a esta situación, se entiende que aunque las 
especies maderables han aportado al desarrollo de las comunidades campesinas y son muy conocidas a nivel local (tanto su nombre y sus usos), a nivel científico estos productos son prácticamente desconocidos, lo que ha ocasionado que en muchos países en desarrollo, como Colombia, halla una pérdida significativa del conocimiento tradicional sobre el uso de estas especies maderables (Benz, CevaIlos, Santana, Rosales, \& Graff, 2000; Katewa, Chaudhary \& Jain, 2004; Córdoba \& García, 2011; Cogollo \& García, 2012).

Del mismo modo, se ha notado que en las comunidades la extracción del recurso maderable se realiza de forma artesanal, sin ningún tipo de mecanización y bajo conocimientos adquiridos empíricamente, efectuados de la siguiente manera: ubicación de área con potencial maderero; limpieza de árboles de lianas y bejucos; tumba, corte, derriba o apeo de árboles; limpieza de fustes, desrame, descope y troceo; labrado de trozas con ayuda de motosierra y/o hacha; apertura de trochas cuando es necesario (Marín, Cárdenas-López \& Suárez-Suárez, 2005). En lo que se refiere al departamento de Chocó, hay que resaltar que no es ajeno a lo descrito anteriormente, debido a que las comunidades asentadas en estos suelos por tradición han utilizado el bosque para el aprovechamiento de manera selectiva, cosechando únicamente las especies maderables de alto valor comercial actual. Esta labor se adelanta mediante las siguientes actividades: selección y/o identificación de los arboles a talar, corte de lianas y bejucos, apeo del árbol, descope, desrame, troceo, descortezado, obtención de los productos, desembosque de la madera y transporte mayor de esta misma (Ramírez \& Ledezma, 2007; Klinger, Ramírez \& Guerra, 2011).

Lo anterior, demuestra que existen una serie de conceptos empíricos claros referidos al aprovechamiento de los recursos maderables del bosque, no obstante, la información disponible crea ciertas incertidumbre en lo que se refiere a las características del aprovechamiento como tal, encontrándose muchas veces restringida por falta de sistematización, lo cual afecta o retrasa el mejoramiento en los procesos productivos adelantados en los bosques del área de estudio. Conscientes y conocedores de esta situación, se realizó la presente investigación, buscando identificar las principales características del aprovechamiento forestal en los municipios de Atrato, Cértegui, Istmina y Medio San Juan del departamento de Chocó, Colombia. Más específicamente se abordaron temas como las especies más aprovechadas en el área de estudio, su transformación, producción por aprovechamiento, forma de comercialización de lo producido, residuos generados en las actividades de corta, conservación del material extraído, usos y costo-beneficio de la cosecha forestal. Toda esta información es útil como punto de partida para la toma de decisiones tendientes al mejoramiento de los procesos productivos a nivel de los ecosistemas forestales.

\section{Metodología}

Área de estudio. El presente estudio se realizó en los municipios de Atrato, Cértegui, Istmina y Medio San Juan (Figura 1), ubicados en el centro sur del departamento del Chocó, Colombia, geográficamente situados a $5^{\circ} 17^{\prime} \mathrm{N}$ y $76^{\circ} 38^{\prime} \mathrm{W}$ y 68 m.s.n.m. Posee una extensión de $4.126 \mathrm{Km}^{2}$ (8,9\% del área departamental), una población de 67.073 habitantes (13,7\% de la población departamental); corresponde a la zona de vida de bosque pluvial tropical (bp-T), que se caracteriza por presentar temperatura promedio de $27^{\circ} \mathrm{C}$, precipitación media de $9.000 \mathrm{~mm}$ anuales (Holdridge, 2000). Entre las actividades económicas desarrolladas en esta zona del departamento sobresale la explotación minera y maderera, agricultura de pancoger y muy poco la pesca (Mosquera, Escobar \& Moreno, 2011). 


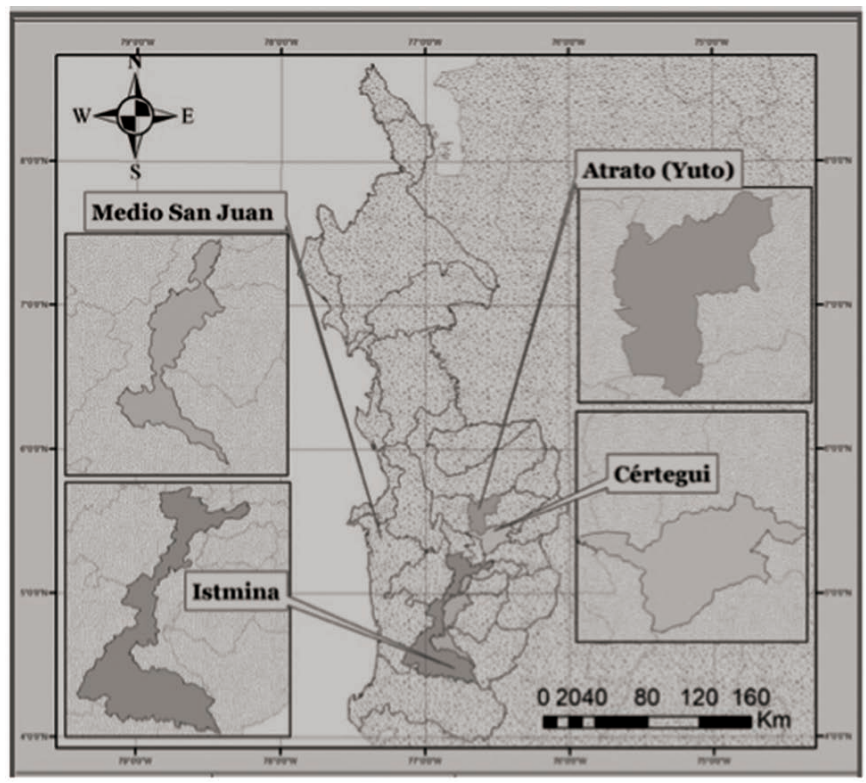

Figura 1. Ubicación del Área de estudio, departamento de Chocó, Colombia

Métodos. El trabajo de campo consistió en encuestar y entrevistar al personal responsable de realizar el aprovechamiento maderero y a las personas que reciben beneficios directos e indirectos de esta misma actividad. Para una mayor organización, la investigación se realizó mediante tres fases a saber:

Primera fase - fundamentación conceptual (Información secundaria). Por medio de una revisión bibliográfica, se recabó información relacionada con los aprovechamientos forestales realizados en el departamento del Chocó. Para esto, se revisaron planes de manejo forestal y documentos de caracterización adelantados en el área de estudio. Esta fase se realizó antes y después de obtener la información primaria en campo.

Segunda fase - recolección de información (Información primaria). Se realizaron recorridos en el área de estudio y se interlocutó con personas de la comunidad que reciben beneficios directos $e$ indirectos del bosque, de acuerdo a la metodología propuesta por los siguientes autores (Ramírez \& Díaz, 2003; Cardona, 2006; López et al., 2007; Medina, Martínez, Barrios \& Bonilla, 2007; Córdoba \& García, 2011; Moreno, Medina, Martínez \& Ruiz, 2013; Torres, Arboleda \& Medina, 2013).
Esta parte de la investigación se enmarco en lo siguiente:

1. Una serie de encuestas estructuradas, las cuales fueron aplicadas a 80 personas (38 operarios de motosierras de los cuatro municipios en estudio, 25 ebanistas "personal de aserrío", 17 personas beneficiarias de las labores de aprovechamiento), con la finalidad de recabar información correspondiente al componente social de las actividades de tala, las especies forestales más aprovechadas en los cuatro municipios, la clase de productos que se obtienen a partir de estas especies, la permanencia de estos productos en el bosque, su conservación y la forma como se comercializan. De igual forma, por medio de la aplicación de las encuestas se recolectó información correspondiente a los costos del aprovechamiento y el valor de los productos obtenidos de las labores de corte. Adicional a lo anterior, se realizaron recorridos al interior del bosque, en los que se observaron de manera detallada las operaciones de corte, desrame, troceo, descortezado y transporte del material talado, con la finalidad de dar respuesta a los siguientes interrogantes: Qué especies se aprovechan con mayor frecuencia (criterios de 
selección de los árboles a aprovechar), cuál es el diámetro mínimo de corta (DMC), la altura del fuste aprovechable, los productos que se obtienen, uso, transporte de las especies, destino de la producción y por último el costo de las actividades productivas.

2. Charlas y Entrevistas. Se sostuvieron conversaciones con las personas beneficiarias del material extraído del bosque, sobresaliendo aquellas que trabajan en los aserríos y las comunidades aledañas a la zona objeto de aprovechamiento forestal, con la finalidad de recabar información que permitiera determinar el uso del listado de especies obtenido mediante la aplicación de las encuestas y la clase de producto elaborado a nivel local.

Tercera fase - Análisis de la información. En esta fase del trabajo se realizó la tabulación e interpretación de la información a través de tablas construidas utilizando el programa Microsoft Excel. Con los datos provenientes de campo se determinó la rentabilidad del aprovechamiento forestal, utilizando la Relación Costo Beneficio (R/B/C)
(Gómez \& Quirós, 2001), la cual se calculó mediante la ecuación 1:

$\mathrm{R} / \mathrm{B} / \mathrm{C}$

\section{Resultados}

\section{Edades y nivel de escolaridad de las personas que realizan el aprovechamiento forestal}

Las personas encuestadas presentaron un rango de edad entre 25 - 61 años, siendo el rango de 30 y 40 años donde se encontró el mayor número de personas que realizan esta actividad, los cuales se dedican en $100 \%$ al aprovechamiento forestal. Además se puede mencionar que las personas entre 20 y 30 años, aunque hacen parte de los procesos productivos, combinan el aprovechamiento forestal con otras actividades (Figura 2A). Por otro lado, se encontró que el nivel de escolaridad de las personas que participan en las actividades de aprovechamiento es muy bajo, donde el máximo nivel alcanzado fue de grado 11 de bachillerato y la mayor parte del personal encuestado solo cursaron entre $1^{\circ}$ - $^{\circ}$ o grado de educación básica. (Figura 2B).
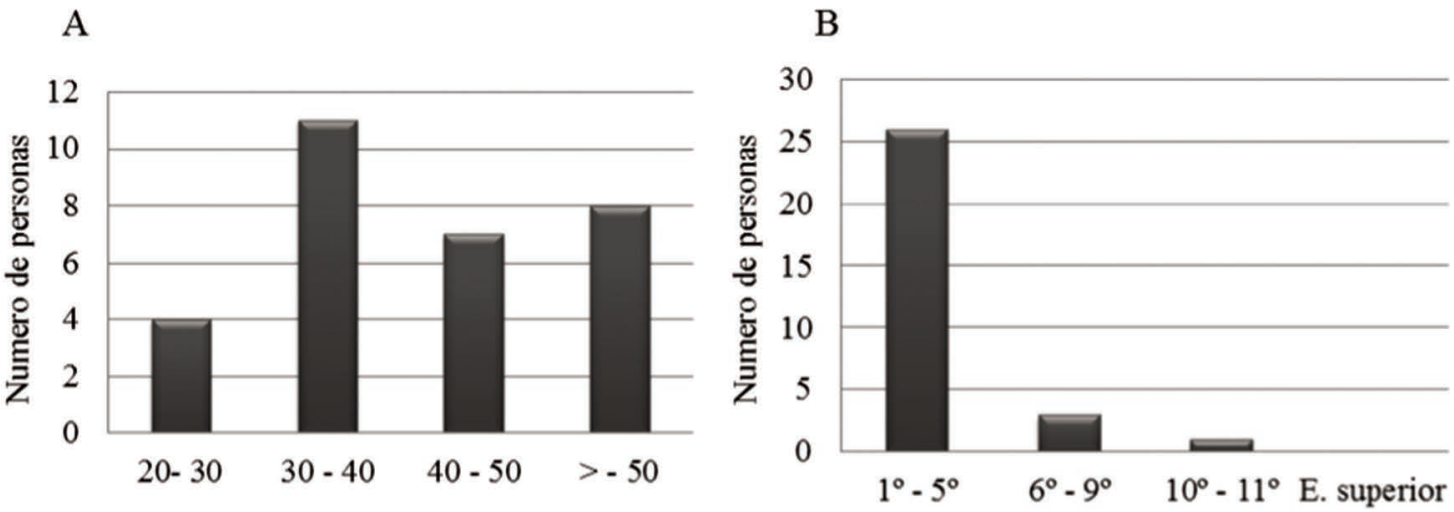

Figura 2. Rango de edad (A) y Nivel de escolaridad de las personas encuestadas (B).

\section{Especies más aprovechadas y principales características del aprovechamiento forestal} Las especies forestales de valor comercial que se aprovechan con mayor frecuencia en los cuatro municipios de estudio son: Lechero (Brosimum utile (Kunth) Oken), Algarrobo (Hymenaea oblongifolia Huber.), Cauchillo (Pseudolmedia laevigata Trecúl), Cedro Macho (Cedrela odorata L.), Lirio (Couma macrocarpa Barb. Rodr.), Aceite (Calophyllum longifolium Willd.), Chanó (Humiriastrum 
procerum Cuatr.), Chachajo (Aniba perutilis Hemsl.) y Caimito (Pouteria amygdalicarpa (Pittier) T.D. Penn.), dado que éstas son las especies con mayor demanda en el mercado regional, además son aquellas de las que se conocen algunos usos a nivel interno de la comunidad (Tablas 1 y 2 ).

El aprovechamiento forestal para estas especies se realiza de forma artesanal, sin ningún tipo de mecanización y bajo conocimientos adquiridos empíricamente, efectuados de la siguiente manera: ubicación de área con potencial maderero; limpieza de árboles de lianas y bejucos; tumbe, corte, derriba o apeo de árboles; limpieza de fustes, desrame, descope y troceo; labrado de trozas con ayuda de motosierra y/o hacha; apertura de trochas cuando es necesario. La tala de árboles de las especies más aprovechadas (Tabla 1) es realizada por cuadrillas de tres personas con una motosierra de referencia STIHL y SUPER STHIL $070 \mathrm{AV}$, con espada de $90 \mathrm{~cm}$ (con la cual se realiza también el descope, desrame, troceo y despunte de los árboles) y/o hacha para la obtención de leña.

Tabla 1. Especies forestales maderables registradas como las más aprovechadas en los cuatro municipios de estudio.

\begin{tabular}{|c|c|c|c|}
\hline \multicolumn{4}{|c|}{ MUNICIPIOS } \\
\hline Atrato & Cértegui & Istmina & Medio San Juan \\
\hline $\begin{array}{l}\text { Couma macrocarpa } \\
\text { Barb. Rodr. }\end{array}$ & $\begin{array}{l}\text { Humiriastrum procerum } \\
\text { Cuatr. }\end{array}$ & $\begin{array}{l}\text { Hymenaea oblongifolia } \\
\text { Huber. }\end{array}$ & Cedrela odorata L. \\
\hline Virola sebifera Aubl. & $\begin{array}{l}\text { Couma macrocarpa } \\
\text { Barb. Rodr. }\end{array}$ & Symphonia globulifera L. & $\begin{array}{l}\text { Brosimum utile (Kunth) } \\
\text { Oken }\end{array}$ \\
\hline $\begin{array}{l}\text { Brosimum utile (Kunth) } \\
\text { Oken }\end{array}$ & $\begin{array}{l}\text { Himatanthus sucuuba } \\
\text { Woodson }\end{array}$ & Protium amplum Cuatrec. & Symphonia globulifera L. \\
\hline $\begin{array}{l}\text { Hymenaea oblongifolia } \\
\text { Huber. }\end{array}$ & Simarouba amara Aubl. & Aniba perutilis Hemsl. & $\begin{array}{l}\text { Hieronyma antioquensis } \\
\text { Cuatrec. }\end{array}$ \\
\hline Inga acrocephala Steud. & $\begin{array}{l}\text { Brosimum utile (Kunth) } \\
\text { Oken }\end{array}$ & $\begin{array}{l}\text { Compsoneura cuatreca- } \\
\text { sasii A.C. Sm. }\end{array}$ & $\begin{array}{l}\text { Pouteria amygdalicarpa } \\
\text { (Pittier) T.D. Penn. }\end{array}$ \\
\hline $\begin{array}{l}\text { Humiriastrum procerum } \\
\text { Cuatr. }\end{array}$ & $\begin{array}{l}\text { Pouteria amygdalicarpa } \\
\text { (Pittier) T.D. Penn. }\end{array}$ & Inga acrocephala Steud. & $\begin{array}{l}\text { Himatanthus sucuuba } \\
\text { Woodson }\end{array}$ \\
\hline Licania micrantha Miq. & $\begin{array}{l}\text { Cespedesia spathulata } \\
\text { (Ruiz \& Pav.) Planch }\end{array}$ & $\begin{array}{l}\text { Garcinia intermedia (Pit- } \\
\text { tier) Hammel }\end{array}$ & $\begin{array}{l}\text { Hymenaea oblongifolia } \\
\text { Huber. }\end{array}$ \\
\hline Dipteryx oleifera Benth. & & $\begin{array}{l}\text { Protium colombianum } \\
\text { Cuatrec. }\end{array}$ & $\begin{array}{l}\text { Tabebuia chrysantha } \\
\text { (Jacq.) S. O. Grose }\end{array}$ \\
\hline $\begin{array}{l}\text { Gustavia excelsa R. } \\
\text { Knuth }\end{array}$ & & Cedrela odorata L. & $\begin{array}{l}\text { Ocotea cernua (Nees) } \\
\text { Mez }\end{array}$ \\
\hline \multirow[t]{2}{*}{$\begin{array}{l}\text { Pouteria amygdalicarpa } \\
\text { (Pittier) T.D. Penn. }\end{array}$} & & $\begin{array}{l}\text { Ocotea cernua (Nees) } \\
\text { Mez }\end{array}$ & $\begin{array}{l}\text { Protium amplum Cua- } \\
\text { trec. }\end{array}$ \\
\hline & & $\begin{array}{l}\text { Hieronyma antioquensis } \\
\text { Cuatrec. }\end{array}$ & \\
\hline
\end{tabular}


El diámetro de corte depende de la utilidad que se le va a dar a la madera, por ejemplo para la obtención de trozas, bloques, soleras, guayacanes, $2 \times 2,2 \times 3$ y tablas se cortan árboles con más de $40 \mathrm{~cm}$ de diámetro, mientras que para la obtención de varetas para andamios o material utilizado en bases y leña no se tiene en cuenta el DMC (incluyendo en estos, individuos de la regeneración natural). Los criterios de selección utilizados para la tala de árboles son: que la especie tenga buena demanda en el mercado, que los árboles sean preferiblemente grandes (con diámetro $\geq 40$ $\mathrm{cm}$ ), rectos y que el lugar donde se encuentran los ejemplares a talar sea de fácil acceso (buscando facilidad para el transporte del material talado).

El número de árboles a aprovechar está determinado por varios factores como: número de personas por grupo de trabajo, herramientas, equipos e insumos a disposición. La distribución de los árboles a ser aprovechados es aislada, por ejemplo en 1 ha pueden aprovecharse entre 10 y 16 árboles de alto valor comercial, cada uno de ellos con una altura comercial promedio de 9 a $16 \mathrm{~m}$, y de cada árbol se pueden aprovechar entre 3 y 5 trozas (una troza tiene 3 a 3,20 $\mathrm{m}$ de largo por aproximadamente $40 \mathrm{~cm}$ de diámetro), es decir, que en una ha se obtiene un promedio de 30 a 80 trozas para ser comercializadas. Cuando el bosque es poco intervenido se pueden aprovechar en promedio 24 árboles ha-1 ${ }^{-1}$ de importancia económica

\section{Uso de las especies más aprovechadas.}

Entre las utilidades que se les da a los productos maderables en los municipios de Atrato, Cértegui, Istmina, y Medio San Juan, sobresalen la construcción de casas, canoas, canaletes y palancas para transporte fluvial; materiales como pilón y mazo para el trillado tradicional de arroz; armarios, camas, sillas y cucharones para el uso doméstico; a parte de esta utilidad se encuentra la medicinal, debido a que, especies como lirio y el palo de perico sirven para el tratamiento de enfermedades como gastritis y malaria respectivamente y de otras especies se utiliza la corteza para baños frescos (Tabla 2).

Tabla 2. Uso de los productos forestales maderables de los cuatro municipios de estudio

\begin{tabular}{|c|c|c|c|}
\hline $\mathrm{N}^{\circ}$ de orden & Nombre común & Nombre Científico & Uso \\
\hline 1 & Caraño & Protium amplum Cuatrec. & Construcción (Formaleta) \\
\hline 2 & Lirio & Couma macrocarpa Barb. Rodr. & $\begin{array}{l}\text { Construcción (Casa), Me- } \\
\text { dicina tradicional (Cura de } \\
\text { la gastritis, mediante el uso } \\
\text { del exudado) }\end{array}$ \\
\hline 3 & Nuanamo & Virola sebifera Aubl. & $\begin{array}{l}\text { Construcción (Casa, } \\
\text { cucharones) }\end{array}$ \\
\hline 4 & Lechero & Brosimum utile (Kunth) Oken & $\begin{array}{l}\text { Construcción (Formaletas } \\
\text { y casas), }\end{array}$ \\
\hline 5 & Algarrobo & Hymenaea oblongifolia Huber. & $\begin{array}{l}\text { Construcción (casa), } \\
\text { Alimentación, Medicina }\end{array}$ \\
\hline 6 & Guamillo & Inga acrocephala Steud & $\begin{array}{l}\text { Combustible (leña) Cons- } \\
\text { trucción (cucharones) }\end{array}$ \\
\hline
\end{tabular}


continuación Tabla 2

\begin{tabular}{|c|c|c|c|}
\hline $\mathrm{N}^{\circ}$ de orden & Nombre común & Nombre Científico & Uso \\
\hline 7 & Chanó & Humiriastrum procerum Cuatr. & $\begin{array}{l}\text { Construcción (Mesa, casa, } \\
\text { bateas, Armario, camas, } \\
\text { etc.) }\end{array}$ \\
\hline 8 & Carbonero & Licania micrantha Miq. & $\begin{array}{l}\text { Construcción (camas, } \\
\text { casa, etc.), combustible } \\
\text { (leña) }\end{array}$ \\
\hline 9 & Choibá & Dipteryx oleifera benth & $\begin{array}{l}\text { Construcción (Canoas, } \\
\text { cama, casa, Armario, pilón } \\
\text { y maso) }\end{array}$ \\
\hline 10 & Pacó & Gustavia excelsa R. Knuth & $\begin{array}{l}\text { Construcción (Formaleta, } \\
\text { casa) }\end{array}$ \\
\hline 11 & Caimito & $\begin{array}{l}\text { Pouteria amygdalicarpa (Pittier) T.D. } \\
\text { Penn. }\end{array}$ & $\begin{array}{l}\text { Construcción (palancas, } \\
\text { casa, canaletes, etc.) }\end{array}$ \\
\hline 12 & Palo Plátano & Himatanthus sucuuba Woodson & $\begin{array}{l}\text { Construcción (Casas, } \\
\text { formaletas) }\end{array}$ \\
\hline 13 & Palo Perico & Simarouba amara Aubl. & Construcción, Medicina \\
\hline 14 & Casaco & $\begin{array}{l}\text { Cespedesia spathulata (Ruiz \& Pav.) } \\
\text { Planch }\end{array}$ & $\begin{array}{l}\text { Construcción (Canoas, } \\
\text { Bateas, casas, mesas, } \\
\text { Armario, pilón y maso) }\end{array}$ \\
\hline 15 & Manchare & Symphonia globulifera L. & Combustible (leña) \\
\hline 16 & Anime & Protium amplum Cuatrec. & $\begin{array}{l}\text { Construcción (canoas, } \\
\text { palanca) }\end{array}$ \\
\hline 17 & Chachajo & Aniba perutilis Hemsl. & $\begin{array}{l}\text { Construcción (Canoas, } \\
\text { palanca) }\end{array}$ \\
\hline 18 & Cuangare & Compsoneura cuatrecasasii A.C. Sm. & $\begin{array}{l}\text { Construcción (Palanca, } \\
\text { casas, canaletes) }\end{array}$ \\
\hline 19 & Aceite & Garcinia intermedia (Pittier) Hammel & $\begin{array}{l}\text { Construcción (casas, } \\
\text { Canoas) }\end{array}$ \\
\hline 20 & Cedro Macho & Cedrela odorata L. & Construcción (Casas) \\
\hline 21 & Jigua Negro & Ocotea cernua (Nees) Mez & $\begin{array}{l}\text { Construcción (Formaletas, } \\
\text { casas) }\end{array}$ \\
\hline 22 & Guayacán & $\begin{array}{l}\text { Tabebuia chrysantha (Jacq.) S. O. } \\
\text { Grose }\end{array}$ & Construcción (casas) \\
\hline 23 & Pantano & Hieronyma antioquensis Cuatrec. & Construcción (Casas) \\
\hline
\end{tabular}




\section{Red de caminos}

En las áreas donde se realiza el aprovechamiento se construyen trochas o mangas, de más de $3 \mathrm{~m}$ de ancho que varían en longitud (50 m - $200 \mathrm{~m}$ ) de acuerdo a la distancia que exista hasta algún cauce bien sea quebrada o río, los cuales en épocas de lluvia aumentan el caudal de forma tal que permiten movilizar la madera en balsas.

Transformación de la madera

El primer grado de transformación de la madera es realizado en el bosque, en el que se obtienen bloques y trozas (Tabla 3). Los aserríos son los encargados del segundo grado de transformación (soleras, $2 \times 2,2 \times 3$, vigas, guayacanes y tablas camas, sillas, armario, muebles, etc.) (Figura 2), sin desconocer que muchas transformaciones se realizan fuera de estos dos espacios, ejemplo la construcción de canoas, bateas, palancas, canaletes, pilón, maso, etc. (construidos por tradición en las zonas rurales, preferiblemente en el sitio donde se apea el árbol) (Tabla 2).

Tabla 3. Producción y forma de comercialización del material extraido del bosque

\begin{tabular}{lccl}
\hline $\begin{array}{c}\text { Material o producto } \\
\text { obtenido }\end{array}$ & $\begin{array}{c}\text { Producción } \\
\left(\mathbf{m}^{3}\right)\end{array}$ & $\begin{array}{c}\text { Volumen } \\
\text { Comercializado }\left(\mathbf{m}^{3}\right)\end{array}$ & Destino \\
\hline Trozas y Bloques & 412 & 284,28 & $\begin{array}{l}\text { Mercado externo (Antioquia, } \\
\text { Risaralda, principalmente) }\end{array}$ \\
& & 127,72 & Mercado Local \\
\hline Total & $\mathbf{4 1 2}$ & \\
\hline
\end{tabular}

Nota: los $127,72 \mathrm{~m}^{3}$ de madera son transformados en vigas, Guayacanes, 2x2, 2x3 y tablas en centros de acopio locales

\section{Proceso de aprovechamiento y forma de comercialización.}

El proceso de aprovechamiento forestal es realizado por cuadrillas de aserradores (tres personas) en periodos de 8 horas diarias, las cuales llegan a obtener producciones de $1,72 \mathrm{~m}^{3}$ por hora, equivalente a 412 $\mathrm{m}^{3}$ en un mes de trabajo (cuando es continuo). De este total, el $69 \%$ es para la producción de trozas y bloques, los cuales son comercializados con intermediario y transportados hacia departamentos como
Antioquia y Risaralda. El 31\% restante es vendido a los centros de acopios locales, quienes lo transforman en $2 \times 2$, Guayacanes, $2 \times 3$, vigas, tablas y soleras (Tabla 3; Figura 3). Los productos como trozas y bloques son apilados en el bosque, en el que permanecen de 3 a 10 días, hasta que son transportados en mulas o a hombro por trochas hasta la vía principal y en balsas (lugares cercanos al rio). 


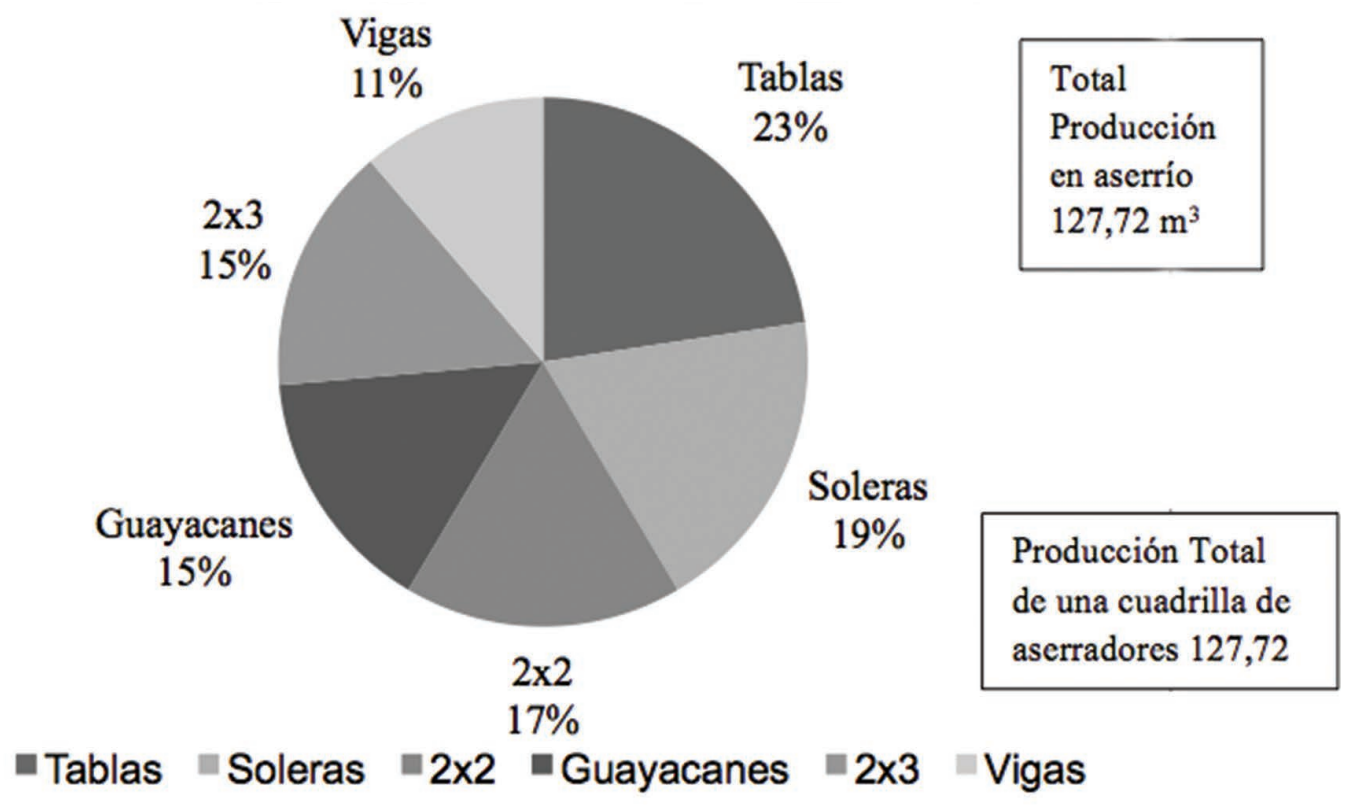

Figura 3. Producción en los centros de acopio locales (aserríos)

\section{Proceso de comercialización de los productos forestales extraídos del bosque.}

El proceso de comercialización de los productos forestales en el área de estudio es muy irregular, dado a que, la extracción de madera solo se realiza cuando es solicitada (depende de la demanda del mercado), queriendo decir con esto, que no existe una producción constante durante todo el año, por lo que los aserradores alternan las actividades de tala con otras actividades como la minería. La comercialización de los productos forestales extraídos del bosque se realiza de dos formas: 1) la adelantada con la intervención de intermediarios (los cuales muchas veces son los que contratan la cuadrilla de aserradores), quienes comercializan los productos dentro y fuera del departamento y 2) mediante el acuerdo entre el aserrador - el dueño del predio y/o de los árboles y el ebanista o dueño de aserradero, en este caso el dueño del aserradero compra la madera en pie al dueño del predio y contrata a un aserrador, quien apea el árbol, lo desrama y lo trocea de acuerdo a las peticiones del solicitante del material maderable (el transporte se acuerda entre aserrador y solicitante).

\section{Residuos generados de las actividades de aprovechamiento maderero.}

Los fustes huecos y torcidos, los que no tienen las dimensiones para ser aprovechados, los árboles que se astillan en la caída, las cantoneras o costeras, las ramas y el aserrín resultante del proceso de aprovechamiento quedan al interior del bosque (no se le da ninguna utilidad), a excepción de algunos lugares en donde se utilizan como puente las cantoneras para cruzar los cursos de agua que se encuentran sobre la red de caminos del bosque y como leña para la cocción de alimentos. La utilidad del aserrín generado en las operaciones de tala depende mucho del lugar donde se realice el aprovechamiento, por ejemplo en los municipios de Atrato y Cértegui el aserrín que queda después del aprovechamiento no es utilizado por la comunidad, porque esto aumentaría los costos de aprovechamiento; por su parte, en los municipios de Istmina y Medio San Juan este aserrín es reutilizable, debiéndose esto, a la cercanía de las casas al bosque, facilitando un poco el transporte de este material que es utilizado en varios caseríos de la cuenca del San Juan como cama de galpones para la cría de pollos y como abono para los cultivos agrícolas (Tabla 4). 
Tabla 4. Utilidad de los residuos generados en las labores de aprovechamiento

\begin{tabular}{ll}
\hline Material Generado (Residuos) & \multicolumn{1}{c}{ Uso y/o Disposición Final } \\
\hline Fustes Huecos & $\begin{array}{l}\text { No se utilizan. Quedan al interior del } \\
\text { bosque }\end{array}$ \\
\hline Fustes Torcidos & $\begin{array}{l}\text { Parte de estos se utilizan como leña } \\
\text { para cocción de alimentos (depen- } \\
\text { diendo la especie y de la cercanía del } \\
\text { lugar donde se adelanta el aprove- } \\
\text { chamiento) }\end{array}$ \\
\hline Árboles sin dimensiones & $\begin{array}{l}\text { No se utilizan. Quedan al interior del } \\
\text { bosque }\end{array}$ \\
Árboles astillados en la caída & $\begin{array}{l}\text { No se utilizan. Quedan al interior del } \\
\text { bosque }\end{array}$ \\
Cantoneras o costeras & $\begin{array}{l}\text { En algunas comunidades se utilizan } \\
\text { como puentes para cruzar los cursos } \\
\text { de aguas presentes en el bosque }\end{array}$ \\
$\begin{array}{ll}\text { En lugares lejanos a los centros po- } \\
\text { blados se dejan al interior del bosque; } \\
\text { mientras que si el aprovechamiento } \\
\text { se realiza cerca de los centros pobla- } \\
\text { dos estas rama se utilizan como leña } \\
\text { para la cocción de alimentos }\end{array}$ \\
de Aprovechamiento & $\begin{array}{l}\text { En lugares lejanos a los centros po- } \\
\text { blados se dejan al interior del bosque; } \\
\text { mientras que si el aprovechamiento } \\
\text { se realiza cerca de los centros pobla- } \\
\text { dos este material se utiliza como sus- } \\
\text { trato para la propagación de hongos y } \\
\text { de especies vegetales }\end{array}$ \\
\hline
\end{tabular}

\section{Conservación de los productos obtenidos del bosque.}

Para la conservación de las tablas se utilizan productos como Furadan, ACPM y aceite quemado, los cuales se aplican una vez seca la madera utilizando brochas (esto se realiza en horas de la mañana). A las trozas, bloques, listones y soleras no se le aplican ninguna clase de conservación.

\section{Costos del aprovechamiento forestal}

Para el análisis costo - beneficio del aprovechamiento forestal, se contó con información suministrada por personas que realizan actividades productivas en los bosques del área de influencia del trabajo. En ese sentido se consideró que a lo largo de una semana un aserrador con un ayudante y un polinero pueden aprovechar 1 ha de bosque (en promedio 15 árboles de alto valor comercial y con D.A.P $\geq 40 \mathrm{~cm}$ ); para lo que se requieren alrededor de $\$ 560.000$ semanales, equivalentes a \$2'820.500 mensuales, sin desconocer que este valor varía de acuerdo con la distancia en la que se encuentra el sitio donde se realiza el aprovechamiento maderero (Tabla 5). 
Tabla 5. Costos de las actividades de aprovechamiento forestal en los municipios de estudio

\begin{tabular}{|c|c|c|c|c|c|c|}
\hline $\begin{array}{c}\text { Grupo } \\
\text { encuestado }\end{array}$ & Actividades & Semanas & $\begin{array}{l}\text { Costo (\$)/ } \\
\text { Semana }\end{array}$ & $\begin{array}{c}\text { Gastos } \\
\text { totales (\$)/ } \\
\text { semana }\end{array}$ & $\begin{array}{c}\text { Costo (\$)/ } \\
\text { mes }\end{array}$ & $\begin{array}{l}\text { Gastos totales } \\
\text { (\$)/mes }\end{array}$ \\
\hline \multirow{5}{*}{1} & Aserrador & 1 & 260.000 & \multirow{5}{*}{560.000} & 1.040 .000 & \multirow{5}{*}{2.540 .000} \\
\hline & Ayudante & 1 & 120.000 & & 480.000 & \\
\hline & Polinero & 1 & 120.000 & & 480.000 & \\
\hline & Transporte & 1 & 60.000 & & 240.000 & \\
\hline & Permiso & & & & 300.000 & \\
\hline \multirow{5}{*}{2} & Aserrador & 1 & 250.000 & \multirow{5}{*}{540.000} & 1.000 .000 & \multirow{5}{*}{2.400 .000} \\
\hline & Ayudante & 1 & 120.000 & & 480.000 & \\
\hline & Polinero* & 1 & 120.000 & & 480.000 & \\
\hline & Transporte ** & 1 & 50.000 & & 200.000 & \\
\hline & Permiso & & & & 240.000 & \\
\hline \multirow{5}{*}{3} & Aserrador & 1 & 300.000 & & 1.200 .000 & \multirow{5}{*}{3.250 .000} \\
\hline & Ayudante & 1 & 180.000 & & 720.000 & \\
\hline & Polinero & 1 & 180.000 & 660.000 & 720.000 & \\
\hline & Transporte & 1 & & & 310.000 & \\
\hline & Permiso & & & & 300.000 & \\
\hline \multirow{5}{*}{4} & Aserrador & 1 & 200.000 & \multirow{5}{*}{480.000} & 800.000 & \multirow{5}{*}{2.140 .000} \\
\hline & Ayudante & 1 & 110.000 & & 440.000 & \\
\hline & Polinero & 1 & 110.000 & & 440.000 & \\
\hline & Transporte & 1 & 60.000 & & 240.000 & \\
\hline & Permiso & & & & 220.000 & \\
\hline
\end{tabular}

Alimentación **

Promedio

$\$ 560.000$

$\$ 2.582 .500$

* Este costo incluye transporte de los productos de las actividades de aprovechamiento maderero **Los gastos de transporte incluyen solo pasaje y transporte de materiales e insumos ida y vuelta *** La alimentación corre por cuenta del personal encargado de las labores del aprovechamiento

\section{Beneficio del aprovechamiento forestal}

Cada cuadrilla cuenta con la capacidad de aprovechar alrededor de 15 árboles semanales, queriendo decir con esto, que el personal especificado en la Tabla 5, puede aprovechar tha semana-1. De estos 15 árboles se obtienen en campo aproximadamente 60 trozas semanales. Este material se vende por pulgadas (\$3.400 en promedio), para 
un valor de $\$ 54.400$ por troza, asumiéndose con esto que en una semana de aprovechamiento se obtienen en promedio $\$ 3.264 .000$, equivalente a $\$ 13.056 .000$ mensuales (Tabla 6). Se debe aclarar que cuando el bosque ha sido poco intervenido y el número de personas encargadas de las labores es mayor se pueden aprovechar aproximadamente 24 árboles de importancia económica en una ha. Al interior de los aserríos se obtienen guayacanes, $2 \times 2,2 \times 3$, vigas, soleras y tablas variando su pecio entre $\$ 72.000$ y $\$$ 312.000 (la docena). Es bueno precisar que los aserradores encuestados extraen tablas para venderlas a los pobladores de las comunidades locales para la construcción de viviendas, pero esto no es constante (Tabla 6).

Tabla 6. Valor de los productos obtenidos en en el primer y segundo grado de transformación de los árboles, en el área de estudio

\begin{tabular}{|c|c|c|c|c|c|c|c|c|}
\hline \multirow{2}{*}{$\begin{array}{c}\text { Transforma- } \\
\text { ción }\end{array}$} & \multirow{2}{*}{$\begin{array}{l}\text { Valor } \\
\text { unidad } \\
\text { (\$) }\end{array}$} & \multirow{2}{*}{$\begin{array}{c}\text { Valor } \\
\text { docena } \\
\text { (\$) }\end{array}$} & \multirow{2}{*}{$\begin{array}{l}\text { Observa- } \\
\text { ciones }\end{array}$} & \multicolumn{3}{|c|}{ Cantidad } & \multicolumn{2}{|c|}{ Ingresos (\$) } \\
\hline & & & & $\begin{array}{l}\text { Por } \\
\text { Árbol }\end{array}$ & Semanal & Mensual & Semanal & Mensual \\
\hline Trozas * & $\$ 54.400$ & $\$ 652.800$ & $\begin{array}{l}\text { Obtenido por } \\
\text { aserradores }\end{array}$ & 4 & 60 & 240 & $\$ 3.264 .000$ & $\$ 13.056 .000$ \\
\hline Bloques * & $\$ 32.000$ & $\$ 384.000$ & $\begin{array}{l}\text { Obtenido por } \\
\text { aserradores }\end{array}$ & 8 & 120 & 480 & $\$ 3.840 .000$ & $\$ 15.360 .000$ \\
\hline Guayacanes * & $\$ 26.000$ & $\$ 312.000$ & $\begin{array}{l}\text { Obtenido } \\
\text { en el aserrío }\end{array}$ & & & & & \\
\hline $2 \times 2$ * & $\$ 6.000$ & $\$ 72.000$ & $\begin{array}{l}\text { Obtenido } \\
\text { en el aserrío }\end{array}$ & & & & & \\
\hline $2 \times 3$ * & $\$ 9.000$ & \$ 108.000 & $\begin{array}{l}\text { Obtenido } \\
\text { en el aserrío }\end{array}$ & & & & & \\
\hline Soleras * & $\$ 19.000$ & $\$ 156.000$ & $\begin{array}{l}\text { Obtenido } \\
\text { en el aserrío }\end{array}$ & & & & & \\
\hline Vigas * & $\$ 19.000$ & $\$ 216.000$ & $\begin{array}{l}\text { Obtenido } \\
\text { en el aserrío }\end{array}$ & & & & & \\
\hline Tablas * & $\$ 9.000$ & $\$ 108.000$ & $\begin{array}{l}\text { Obtenido } \\
\text { en el aserrío }\end{array}$ & & & & & \\
\hline
\end{tabular}

* Datos promedios; su valor puede aumentar o disminuir de acuerdo con la calidad de la madera

Relación Costo - Beneficio (R/B/C).

De acuerdo con los datos de campo se obtuvo una Relación Costo/Beneficio de 6, para las actividades del aprovechamiento maderero realizadas semanal y mensualmente en los municipios de Atrato, Cértegui, Istmina y Medio San Juan, lo que significa que por cada peso que se invierte en esta actividad se obtienen 6 pesos a cambio, indicando esto, que esta actividad económica es rentable.

\section{Discusión}

Especies maderables más aprovechadas de los cuatro municipios en estudio.

Las especies reportadas como más aprovechadas el $B$. utile y el $H$. oblongifolia, se encuentran en el listado de especies más aprovechadas en la sub región del Atrato, Chocó (IDEAM, 2009, Bonilla, Cuesta-Córdoba \& Valois-Cuesta., 2011, 
Moreno et al., 2013); lo que muestra por un lado, la amplia dispersión de las especies en el territorio chocoano, pero al mismo tiempo, preocupa porque al aumentar el número de aprovechamiento sobre estos ecosistemas se reduciría la posibilidad de encontrarla en este territorio (Ramírez \& Ledezma, 2007).

\section{Características del aprovechamiento forestal en el área de estudio.}

El aprovechamiento maderero en bosques de la selva chocoana (especialmente el de los cuatro municipios estudiados), involucra varios aspectos a tener en cuenta. Entre estos se pueden citar la extracción ilegal de material maderable de los bosques (cada 1 de $3 \mathrm{~m}^{3}$ de madera se extrae de forma ilegal) (Ramírez \& Ledezma, 2007); la ubicación de los bosques (difícil acceso al área de aprovechamiento), que de cierta manera condicionan la realización de las actividades de apeo, desrame, descortezado, troceo y transporte del material maderable; el proceso de aprovechamiento se realiza de forma convencional, utilizando motosierra y hacha; el material aserrado es transportado a hombro o por tracción animal, siendo estas características similares a las reportadas por Bonilla et al., (2011), en bosques de San Isidro, municipio de Atrato.

Los aserradores son los encargados del primer eslabón de la cadena de aprovechamiento forestal, la cual consiste en la realización de las actividades de apeo, descope, desrame, descortezado, troceo y transporte de la madera hasta la orilla del camino o vía principal. Referente a esto el IDEAM (2009), expresa que el ingreso económico obtenido por la realización de las actividades descritas con anterioridad es demasiado bajo, obteniéndose un $10 \%$ de todo el proceso de aprovechamiento. El mismo autor atribuye esto al hecho de que los aserradores acuerdan el precio de la madera con los intermediarios y dueños de la madera, los cuales, se quedan con gran parte de las ganancias del proceso de aprovechamiento.

\section{Transformación y Uso.}

Localmente se realizan dos eslabones de la cadena de aprovechamiento, el primero que es efectuado por los aserradores (obteniendo trozas, bloques y tablas) y el segundo adelantado por los propietarios de aserraderos o ebanistas que trabajan con productos primarios extraídos del bosque, obteniendo muebles, camas, armarios, nocheros de madera fina para la venta local específicamente, al respecto el IDEAM (2009), sugiere que para hacer del aprovechamiento un motor de desarrollo de departamentos como el Chocó, se debe incluir a nivel local al menos un eslabón más al sistema de aprovechamiento maderero, siendo éste la transformación final (comerciante mayorista), el cual dispone de establecimientos comerciales en los cuales se venden machimbre, molduras, guarda escobas y productos acabados como muebles, tableros y contrachapados.

\section{Producción por aprovechamiento y forma de comercialización de lo producido.}

En las actividades de aprovechamiento forestal realizadas en el área de estudio una cuadrilla de aserradores obtiene $1,72 \mathrm{~m}^{3} \mathrm{~h}^{-1}$; este valor es menor al reportado por Turc \& Mazzuco (1998) en bosques santiagueños de Argentina $\left(2,50 \mathrm{~m}^{3} \mathrm{~h}^{-1}\right)$, estas diferencias se deben en gran medida a la presentación final de los productos que se extraen del bosque, por ejemplo en lo de Santiago, Argentina se obtienen madera rolliza, mientras que en el área de estudio se extraen trozas, bloques, listones, solera, $2 \times 2,2 \times 3$, guayacanes y tablas principalmente, lo que genera mayores porcentajes de desperdicios al pasar de una transformación a otra (Medina et al., 2007; Moreno et al., 2013).

\section{Residuos generados de las actividades de aprovechamiento maderero.}

Otra de las características del aprovechamiento maderero en el área de estudio es sin duda, la generación de desperdicio o residuos. Entre estos, sobresale el aserrín, el cual es utilizado en la producción de biogás y como sustrato orgánico para la propagación de hongos y especies vegetales (Mosquera, Escobar \& Moreno, 2011; Aguilar, Martínez \& Ríos, 2009; Martínez, Medina \& Ríos, 2010). En lo referente al uso, el aserrín es el material que 
se reutiliza, especialmente, en los municipios de Istmina y Medio San Juan, aclarando que parte de este material que no alcanza a ser utilizado se deposita en los cursos de agua, información que es similar a la reportada por Moreno et al. (2013), en el municipio de Quibdó, por lo que este mismo autor sugiere ampliar el uso que se le da a este material en la cadena de aprovechamiento maderero.

Krueger (2003) y Medina et al. (2007), atribuyen los elevados valores de desperdicios en las actividades de aprovechamiento a la destreza de los aserradores a la hora de realizar los cortes (astillamiento de los árboles al momento de la caída y dimensiones insuficientes de diámetro y altura a la hora de realizar el corte). De acuerdo con Medina et al. (2007), las labores en el bosque llegan a generar desperdicios superiores al $80 \%$, tomando como referencia el volumen total del árbol en pie. Estos autores, expresan que el porcentaje de desperdicio en las labores de aprovechamiento maderero tiende a aumentar con el grado de transformación, obteniéndose valores de 39,96\% para el volumen comercial, $70,76 \%$ para las trozas, $86,41 \%$ para los bloques y $91,34 \%$ para el material procesado. Del mismo modo, Moreno et al, (2013), exponen un porcentaje de desperdicio de $45 \%$ en los aserraderos. Estos desperdicios son producto de la deficiencia de formas de los árboles (bifurcaciones), las dimensiones insuficientes y el astillamiento de árboles al momento de la caída (Amaral et al., 2000; Krueger, 2003; Medina et al., 2007).

Según el número de personas encuestadas en esta investigación y teniendo en cuenta las especies registradas para los cuatro municipios, se demuestra que existe un buen nivel de conocimiento de las especies de esta zona del territorio chocoano y la utilidad que se le da a estas mismas, pero estudios donde se evidencien estas temáticas son escasos y más aquello donde se enmarca la transformación y el transporte local de los productos obtenidos del material extraído del bosque, por lo que es necesario adelantar investigaciones, las cuales posibiliten el conocimiento y aprovechamiento integral de estos recursos forestales.

\section{Conclusiones}

En cuanto al potencial existente de los productos forestales maderables (PFM), de acuerdo con esta investigación y experiencias locales, se aprecia que las especies forestales maderables juegan un papel importante en la subsistencia de las comunidades asentadas en los municipios de Atrato, Cértegui, Istmina y Medio San Juan, Io cual se puede observar a lo largo de la cadena de transformación, uso y comercialización de dichos productos, los cuales de forma directa e indirecta generan empleos ocasionales a los habitantes de esta región, por lo que las autoridades gubernamentales se deben enfocar al fortalecimiento de estas actividades y de aquellas especies que se encuentran bajo aprovechamiento comercial no planificado, para que de esta manera se aporte a la sostenibilidad de esta actividad económica.

El aprovechamiento maderero en los municipios estudiados se realiza de forma selectiva, utilizándose como principal herramienta de apeo la motosierra, con la que se adelanta el primer eslabón de la cadena de aprovechamiento (apeo, descope, desrame, descortezado y troceo), en el cual se obtienen productos como trozas, bloques, listones, vigas y tablas. El transporte de este material es realizado a hombro o con tracción animal.

Los ebanistas o dueños de aserríos de la región son los encargados de realizar el segundo grado de transformación al material extraído del bosque, obteniendo sillas, muebles, armarios, cama, entre otros productos. También en la mayoría de los caso, éstos se encargan de la contratación de la cuadrilla de aserradores (hacen las veces de intermediarios).

Los principales usos que se le dan al material forestal maderable extraído del área de estudio son el de construcción y el medicinal. Adicional a esto, a nivel interno de las comunidades se utilizan algunas de las especies reportadas en este estudio para la construcción artesanal de canoas, bateas y pilones para el trillado tradicional del arroz. 
La conservación de los productos extraídos del bosque se realiza utilizando, ACPM, aceite quemado y Furadan, sin desconocer que en las comunidades rurales de estos municipios se tiene entendido que la mejor forma de conservar la madera es dejándola secar al aire libre, ya que muchos no utilizan productos para la conservación y preservación de la madera.

En promedio en el área de estudio se aprovechan 15 árboles ha-1 semanalmente, de los cuales se obtienen alrededor de 60 trozas y 120 bloques.

El aprovechamiento maderero realizado en los municipios de Atrato, Cértegui, Istmina y Medio San Juan es rentable, dado a que por cada peso que se invierte, a cambio se obtienen entre 6 y 7 pesos.

\section{Literatura citada}

1. Aguilar, Y. E., Martínez, M. G. \& Ríos, H. A. (2010). Aprovechamiento de residuos sólidos en algunos municipios del departamento del Chocó. Reunión Nacional ACOFI 2010. El compromiso de las facultades de Ingeniería en la formación, para el desarrollo regional. Septiembre 15 al 17. Santa Marta. 102 p.

2. Amaral, P., Verissimo, A., Barreto, P., Vidal, E., Holdsworth, A., Uhl, C., Isller, F., Zweede, J. \& Buschbacher, R. (2000). Bosques para siempre: Manual para la producción de madera en la Amazonía. Cali, Colombia. 161 p.

3. Arias, J. (2007). Oferta de productos forestales maderables y no maderables con potencial económico en un bosque de tierra firme de la Amazonia colombiana. Bogotá. D.C. Colombia. Recuperado de: http://www.bdigital.unal.edu.co/3384/25/9789587018547_Parte11.pdf

4. Benz, F.; Cevallos, J.; Santana, F.; Rosales, J. \& Graff, M. (2000). La pérdida del conocimiento sobre el uso de plantas en la sierra de la reserva de Biosfera de Manatlàn, México. Recuperado de: http://people.brandeis.edu/orgodoy/ www.northwestern.edu/andthropology/LHBR/Bolivia.html

5. Bonilla, L. N., Cuesta-Córdoba, H. \& Valois-Cuesta, H. (2011). Efectos de la extracción forestal sobre la estructura y composición de un bosque pluvial del Pacífico colombiano. Rev. Biodiversidad Neotropical. 1 (1): 48-54.

6. Cardona, A. (2006). Caracterización de los productos naturales no maderables en el municipio de lloró (chocó), Colombia. Centro de investigaciones y estudios en biodiversidad y recursos genéticos (CIEBREG). Recuperado de: http://es.scribd.com/doc/57944847/ Investigaciones-de-Productos-Forest-Ales-NoMaderables-PFNM-Registrados-en-La-Base-de-Datos-Del-Humboldt.
7. Cogollo, A. M. \& García, C. F. (2012). Caracterización etnobotánica de los productos forestales no maderables (PFNM) en el corregimiento de Doña Josefa, Chocó, Colombia. Revista Biodiversidad Neotropical. 2 (2): 102-112.

8. Córdoba, T. L. \& García, C. F. (2011). Inventario Etnobotánico de especies Frutales Silvestres Comestibles en el municipio de Lloró, Chocó, Colombia. Revista Investigación, Biodiversidad y Desarrollo. 30 (1): 23-31.

9. Challenger, A. \& Soberón, J. (2008). Los ecosistemas terrestres, en Capital natural de México, vol. I: Conocimiento actual de la biodiversidad. Conabio, México, pp. 87-108. Recuperado de: http://www.biodiversidad. gob.mx/pais/pdf/CapNatMex/Vol\%20I/I03_Losecosistemast.pdf

10. Gómez, M. \& Quirós, D. (2001). Análisis financiero del manejo de bosques. p 229 - 263. En: Louman, B.; Quirós, D. \& Nilson, M. (eds.). 2001. Silvicultura de bosques latifoliados húmedos con énfasis en América Central. Turrialba, C. R. 265 p.

11. González, D. V. (2003). Los productos Naturales no maderables (PNNM): Estado del arte de la investigación y otros aspectos. Biocomercio sostenible, Instituto de Investigaciones de recursos Biológicos "Alexander Von Humboldt". Bogotá Colombia. Recuperado de: http:// cdam.minam.gob.pe/publielectro/biocomercio/productosnaturalesnomaderables.pdf.

12. Guariguata, M. R., Fernandez, C. G., Nasi, R., Sheil, D., Jauregui, C. H., Cronkleton, P., Ndoye, O. \& Ingram, V. (2009). Hacia un manejo múltiple en bosques tropicales: Consideraciones sobre la compatibilidad del manejo de madera y productos forestales no maderables. CIFOR, Bogor, Indonesia. Recuperado de http:// www.cifor.org/publications/pdf_files/Books/BGuariguata0901.pdf

13. Holdridge, L. R. (2000). Ecología basada en Zonas de vida. San José: Instituto Interamericano de Cooperación para la Agricultura (IICA). 143 p.

14. IDEAM (Instituto de Hidrología, Meteorología y Estudios Ambientales). (2009). Diseño y puesta en marcha del instrumento de captura de datos (subregistro) e información generada por actividades informales en los procesos de extracción, transformación y comercio de productos forestales. Bogotá D.C. 183 pg.

15. Katewa, S., Chaudhary, B. \& Jain, A. (2004). Bases populares de plantas medicinales de la zona Tribal de Rajastan, India. Ethnopharmacology. 92: 41-46.

16. Klínger, B.W., Ramírez, M. G. \& Guerra, G. J. (eds.). (2011). Aportes al conocimiento de los ecosistemas estratégicos y las especies de interés especial del Chocó Biogeográfico parte I. Instituto de Investigaciones Ambientales del Pacifico (IIAP). Santiago de Cali, Colombia. $158 \mathrm{p}$.

17. Krueger, W. (2003). Efecto del marcado de árboles de futura cosecha y la planificación de pistas de arrastres en el aprovechamiento convencional con limites diamétricos en un bosque tropical de Bolivia. Proyecto de manejo forestal sostenible BOLFOR. Documento Técnico 119/2003; 1-26. Recuperado de http://pdf.usaid.gov/ pdf_docs/Pnacw377.pdf 
18. López, C. R.; Navarro J. A.; Montero M. I.; Amaya-Vecht, K.; Rodríguez-Castañeda, M. \& Polanía-Barbosa, A. (2007). Algunas especies no maderables del corregimiento de Tarapacá (Amazonas), Colombia. Instituto amazónico de investigaciones científicas (SINCHI). Recuperado de: http://es.scribd.com/doc/57944847/ Investigaciones-de-Productos-Forest-Ales-No-Maderables-PFNM-Registrados-en-La-Base-de-Datos-DelHumboldt

19. Marín, C. C., Cárdenas-López, D. \& Suárez-Suárez, S. (2005). Utilidad del valor de uso en etnobotánica: estudio en el departamento de Putumayo (Colombia). Caldasia 27(1):89-101. Recuperado de: http://search. proquest.com/docview/1677413823/fulltextPDF/51F4C AD3CF5A461BPQ/1 ?accountid=37386

20. Martínez, G. M., Medina, H. H. \& Ríos, H. A. (2010). Aprovechamiento de residuos sólidos: Avances en investigaciones realizadas en la Universidad Tecnológica del Chocó. Investigación, Biodiversidad y Desarrollo. 29 (2): 177-185.

21. Medina, H. H., Martínez, G. M., Barrios, M. F. \& Bonilla, J. A. 2007. Determinación del porcentaje de desperdicio en las labores de Aprovechamiento forestal en un bosque pluvial tropical en el municipio de Medio San Juan, Chocó, Colombia. NOVA - Publicación científica en Ciencias Biomédicas. 5 (8): 101-112. Recuperado de: http://www.unicolmayor.edu.co/invest_nova/NOVA/ nova8_artorig5.pdf

22. Moreno, Y. E., Medina, H. H., Martínez, G. M. \& Ruiz, B. A. (2013). Procesos productivos en los centros de acopio (Aserríos) del municipio de Quibdó, Chocó. p 85. En: Memorias del IV Seminario Internacional de
Agroforestería "Desde el Chocó Biogeográfico para el Mundo". Universidad Tecnológica del Chocó. Quibdó, Chocó, Colombia. 17 - 20 de septiembre.

23. Mosquera, A. D., Escobar, D. R. \& Moreno, A. M. (2011). Estructura y función de los huertos caseros de las comunidades afrodescendientes asentadas en la cuenca del río Atrato departamento del Chocó, Colombia. Biodiversidad Neotropical. 1 (2): 91-97.

24. Ramírez, A. \& Díaz, B. (2003). Acercamiento a la identificación de los principales productos forestales no maderables (PFNM) usados en el departamento de Cundinamarca. Universidad Distrital Francisco José de Caldas, Bogotá, Colombia. Recuperado de: http:// es.scribd.com/doc/57944847/Investigaciones-de-Productos-Forest-Ales-No-Maderables-PFNM-Registrados-en-La-Base-de-Datos-Del-Humboldt

25. Ramírez, M. G. \& Ledezma-Rentería, E. (2007). Efecto de las actividades Socioeconómicas (Minería y explotación madrera) sobre los bosques del departamento del Chocó. Revista Institucional de la Universidad Tecnológica del Chocó. (26): 58-65.

26. Torres, J. J.; Arboleda, L. E.; Medina, H. H. (2013). Caracterización de la cadena de transformación, uso y comercialización de productos forestales maderables en cuatro municipios del departamento del Chocó, Colombia. p 42. En: Memorias del IV Seminario Internacional de Agroforestería "Desde el Chocó Biogeográfico para el Mundo". Universidad Tecnológica del Chocó. Quibdó, Chocó, Colombia. 17 - 20 de septiembre.

27. Turc, C. O. \& Mazzucco R. V. (1998). Caracterización de los sistemas de aprovechamiento forestal utilizados en los montes santiagueños. Quebracho. (6): 59 - 68.

\section{Conflicto de Intereses}

Los autores declaran no tener ningún conflicto de intereses 